\title{
Anafilaxia à gentamicina endovenosa: Um caso clínico
}

\section{Anaphylaxis to intravenous gentamicin: A case-report}

Data de receção / Received in: 07/12/2018 Data de aceitação / Accepted for publication in: 07/01/2019

\author{
Rev Port Imunoalergologia 2019;27 (2): I 47-I50
}

Marta Martins, Sofia Farinha, Bárbara Cardoso, Rute Reis, Filipe Inácio

Serviço de Imunoalergologia, Hospital São Bernardo, Setúbal

\section{RESUMO}

A gentamicina, antibiótico aminoglicosídeo, é muitas vezes utilizada como antibioterapia profilática em cirurgia ortopédica. As reações adversas aos aminoglicosídeos são maioritariamente tóxicas, sendo a ototoxicidade e a nefrotoxicidade as mais comuns. No que respeita a reações de hipersensibilidade, são mais frequentes as reações tardias, tipo IV, relacionadas com o uso de preparações oftálmicas ou cimento ósseo contendo gentamicina. No entanto, apesar de raras, estão descritas reações de hipersensibilidade imediata à gentamicina. Apresenta-se o caso clínico de uma doente com reação anafilática após administração endovenosa de gentamicina como profilaxia antibiótica em cirurgia ortopédica. $\mathrm{O}$ estudo alergológico por testes cutâneos com gentamicina confirmou o diagnóstico.

Palavras-chave: Anafilaxia gentamicina, hipersensibilidade lgE-mediada, reações de hipersensibilidade a antibióticos não betalactâmicos.

\section{ABSTRACT}

Gentamicin, an aminoglycoside antibiotic, is often used as prophylactic antibiotic therapy in orthopedic surgery. Adverse reactions to aminoglycosides are mostly toxic, with otoxicity and nephrotoxicity being the most common. As regards to hypersensitivity reactions, late type IV reactions, related to the use of ophthalmic preparations or gentamicin-containing bone cement, are more commonly described. Although rare, there are immediate hypersensitivity reactions to gentamicin. The clinical case of a patient with anaphylactic reaction after intravenous administration of prophylactic gentamicin, in the context of orthopedic surgery, is described. In this case, the skin tests proved useful to the diagnosis.

Keywords: Anaphylaxis gentamicin, IgE-mediated hypersensitivity, hypersensitivity reactions to non-beta-lactam antimicrobial agents. 


\section{INTRODUÇÃO}

\section{A} gentamicina pertence à classe dos antibióticos aminoglicosídeos, ativos contra os Staphylococcus e os bacilos aeróbios gram-negativos, como Escherichia coli, Enterobacter, Klebsiella, Proteus e Pseudomonas. Nas endocardites por Streptococcus e Enterococcus, a gentamicina também é utilizada, combinada com a benzilpenicilina ou a amoxicilina.

Os aminoglicosídeos apresentam baixas resistências microbianas, pelo que são uma ferramenta útil na prática clínica'. Esta classe de antibióticos é com frequência usada em termos empíricos nos casos de infeções graves, como nas septicemias, nas infeções respiratórias nosocomiais, bem como nas infeções urinárias e intra-abdominais graves ${ }^{2}$. São ainda usadas como agentes de segunda linha na profilaxia cirúrgica ${ }^{2}$, sendo que a gentamicina é usada via sistémica, nomeadamente como terapêutica profilática em cirurgia ortopédica ${ }^{3}$.

As reações adversas classicamente descritas com os aminoglicosídeos são a nefrotoxicidade e a ototoxicidade, sendo mais raras as reações de hipersensibilidade ${ }^{2}$. De entre os casos descritos de reações de hipersensibilidade, as mais frequentes são não imediatas, como a dermatite de contacto, sobretudo associada à ampla utilização tópica destes antibióticos (como a neomicina). Alguns estudos mostram também uma importante prevalência de sensibilização a gentamicina em doentes com otite externa crónica, associada ao seu uso tópico ${ }^{4}$.

Outras reações cutâneas não imediatas com aminoglicosídeos têm sido descritas, como o exantema maculopapular, a necrólise epidérmica tóxica (TEN) e a síndrome de hipersensibilidade a fármacos com eosinofilia e sintomas sistémicos (DRESS). '

De entre as reações imediatas aos aminoglicosídeos, a mais descrita é a urticária, sendo raríssimos os casos de anafilaxia publicados. Estão descritos apenas alguns casos de anafilaxia à estreptomicina, presente em meios celulares utilizados em procedimentos de fertilização in vitro, em alimentos ou por absorção cutânea em doentes com eczema de contacto'. São também raros os casos de anafilaxia à gentamicina, estando apenas descritos na literatura 5 casos, um dos quais após administração intramuscular, os restantes por administração endovenosa. Nestes doentes, a positividade dos testes cutâneos à gentamicina (por picada e/ou intradérmicos, nas concentrações utilizadas pelos autores) permitiu confirmar um mecanismo lgE mediado, sendo que em dois dos casos relatados parece coexistir uma sensibilização tipo IV, pela positividade nos testes epicutâneos para a gentamicina e outros aminoglicosideos $5,6,7,8,9$.

A indisponibilidade de concentrações validadas para testes cutâneos a aminoglicosídeos pode dificultar a confirmação diagnóstica.

\section{CASO CLÍNICO}

Doente do sexo feminino, 53 anos, caucasiana, com antecedentes de hipertensão arterial, obesidade, síndrome depressiva e neoplasia da mama (submetida a mastectomia, quimioterapia e radioterapia). Foi referenciada à consulta de Alergia Medicamentosa pela Cirurgia Geral três anos após episódio suspeito de alergia à gentamicina, em contexto de cirurgia ortopédica por fratura do rádio direito: apresentou quadro de hipotensão e eritema generalizado 5 minutos após administração endovenosa deste antibiótico. $O$ quadro reverteu com corticoide e anti-histamínico endovenosos e adrenalina intramuscular. Não se recordava de exposições prévias a gentamicina. Nesta cirurgia foram ainda utilizados os seguintes fármacos: bupivacaína, sufentanil, esomeprazol, diazepam, paracetamol, tramadol e metoclopramida.

A avaliação alergológica foi realizada através de testes cutâneos por picada e intradérmicos e teste de ativação de basófilos com gentamicina. Não foi possível realizar o doseamento de IgE específica sérica de gentamicina, dado esta não estar disponível. Foram excluídos como suspeitos da reação descrita os restantes 
fármacos utilizados durante a cirurgia, uma vez que houve utilização posterior dos mesmos, nomeadamente em contexto cirúrgico (mastectomia e cirurgia reconstrutiva mamária).

Uma vez que não existem concentrações validadas para os testes cutâneos à gentamicina, respeitamos as concentrações máximas não irritativas já reportadas por outros autores ${ }^{7,8,9}$. As concentrações máximas de gentamicina utilizadas foram testadas em quatro controlos, sendo os resultados negativos.

Da avaliação alergológica realizada destacam-se os seguintes resultados: teste intradérmico (ID) na concentração de $4 \mathrm{mg} / \mathrm{mL}$ de gentamicina com leitura aos 20 minutos positiva (Figura I); o teste de ativação dos basófilos a gentamicina foi inconclusivo por ativação basal elevada (Quadro 2).

Quadro I. Testes cutâneos com gentamicina

\begin{tabular}{|c|c|c|}
\hline Concentração & $\begin{array}{c}\text { Prick } \\
\text { (pápula, } \mathrm{mm} \text { ) }\end{array}$ & $\begin{array}{l}\text { Intradérmico } \\
\text { (aumento da } \\
\text { pápula, mm) }\end{array}$ \\
\hline $\begin{array}{l}\text { Controlo } \\
\text { negativo } \\
\text { (NaCL) }\end{array}$ & 0 & - \\
\hline $\begin{array}{l}\text { Controlo positivo } \\
\text { (histamina) }\end{array}$ & $6 \mathrm{~mm}$ & - \\
\hline $0.04 \mathrm{mg} / \mathrm{mL}$ & - & $0 \mathrm{~mm}$ \\
\hline $0.4 \mathrm{mg} / \mathrm{mL}$ & - & $0 \mathrm{~mm}$ \\
\hline $4 \mathrm{mg} / \mathrm{mL}$ & - & $12 \mathrm{~mm}$ \\
\hline $40 \mathrm{mg} / \mathrm{mL}$ & $0 \mathrm{~mm}$ & - \\
\hline
\end{tabular}

Quadro 2. Teste de ativação dos basófilos (TAB)

\begin{tabular}{|l|c|}
\hline \multicolumn{1}{|c|}{ Estímulo } & Gentamicina \\
\hline Concentração & $\mathrm{I}, 2 \mathrm{mg} / \mathrm{mL}$ \\
\hline Ativação & Não validável \\
\hline Índice estimulação & Não validável \\
\hline
\end{tabular}

Nota: Elevada estimulação basal que não permite a validação do teste.

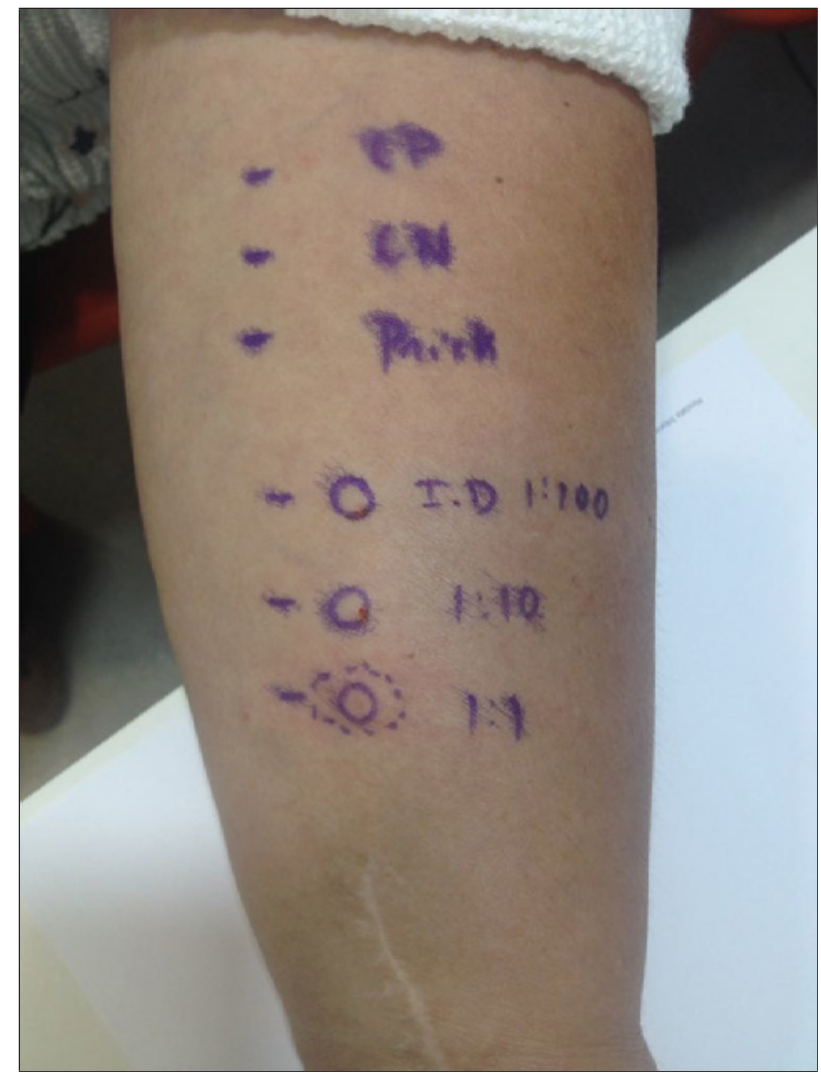

Figura I. Testes cutâneos gentamicina

Da investigação alergológica realizada concluiu-se alergia IgE mediada à gentamicina, tendo em conta que para além da positividade do teste intradérmico a este fármaco os restantes fármacos foram excluídos como suspeitos, dado terem sido utilizados posteriormente à reação com tolerância.

\section{DISCUSSÃO}

Existem poucos casos descritos na literatura de reações de hipersensibilidade mediadas por lgE à gentamicina endovenosa, sendo contudo importante considerar esta hipótese diagnóstica.

Para os aminoglicosídeos, como para a maioria dos antibióticos não betalactâmicos, as concentrações a utilizar nos testes cutâneos não se encontram validados'. 
Existem alguns trabalhos que apresentam valores de concentrações máximas não irritativas para os testes em picada e intradérmicos ${ }^{10}$. No caso apresentado, bem como nos casos anteriormente descritos, os testes cutâneos revelaram-se úteis no diagnóstico.

Será importante continuar a estudar os doentes com suspeita de alergia a aminoglicosídeos, de forma não só a confirmá-la ou excluí-la, mas também na tentativa de validação dos meios complementares de diagnóstico.

Alertamos ainda para a existência, embora rara, de alergia a este grupo de fármacos, por vezes com manifestações graves.

\section{Conflito de interesses}

Os autores declaram que não existem conflitos de interesse.

\section{Contacto:}

Marta Martins Batista

Serviço de Imunoalergologia

Hospital São Bernardo

R. Camilo Castelo Branco 2910-446 Setúbal

E-mail: marttamarttins@gmail.com

\section{REFERÊNCIAS}

I. Sánchez-Borges M, Thong B, Blanca M, Ensina LF, González-Diaz S, Greenberger PA, et al. Hypersensitivity reactions to non beta-lactam antimicrobial agents, a statement of the WAO special committee on drug allergy. World Allergy Organ J 2013;6:18.

2. Poulikakos P, Falagas M.E. Aminoglycoside therapy in infectious diseases. Expert Opin. Pharmacother 2013;14:1585-97.

3. Dubrovskaya Y, Tejada R, Bosco J, Stachel A, Chen D, Feng M, et al. Single high dose gentamicin for perioperative prophylaxis in orthopedic surgery: Evaluation of nephrotoxicity. SAGE open medicine. 2015;3:2050312115612803.

4. Lippo J, Lammintausta K. Positive patch test reactions to gentamicin show sensitization to aminoglycosides from topical therapies, bone cements, and from systemic medication. Contact Dermatitis 2008;59:268-72.

5. Hall F. Anaphylaxis after gentamicin. Lancet 1977; 2(8035):455.

6. Schulze S, Wollina U. Gentamicin-induced anaphylaxis. Allergy 2003; 58:88-9.

7. Connolly M, McAdoo J, Bourke JF. Gentamicin-induced anaphylaxis. Ir J Med Sci 2007;176:317-8.

8. Henao C, Morales C, Villa R, Henao A. Gentamicin-induced anaphylaxis, a case report. World Allergy Organ J 2015;8:A269.

9. Christiansen IS, Pedersen P, Krøigaard M, Mosbech H, Garvey LH. Anaphylaxis to intravenous gentamicin with suspected sensitization through gentamicin-loaded bone cement. J Allergy Clin Immunol Pract 2016;4:1258-9

10. Empedrad RB, Darter AJ, Earl HS, Gruchalla RS. Non-irritating intradermal skin test concentration for commonly prescribed antibiotics. J Allergy Clin Immunol 2003; I 2:629-30. 